\section{African fishes}

$S_{I R}-$ Meyer et al. conclude $^{1}$ that morphological diversification in flocks of cichlid fishes in Lake Victoria occurs without much molecular evolution. This agrees with our own data on hybrids between different species of cichlid fish from Lake Victoria, which can be bred easily over several generations $\mathrm{s}^{2-4}$. In addition to the molecular data on mitochondrial DNA, our results show the genetic compatibility for the entire genome of some Lake Victoria 'haplochromines'.

But we disagree with Meyer et al.'s second conclusion that Lake Malawi species are more closely related to those of Lake Victoria than to those of Lake Tanganyika. We tested by hybridization the genetic compatibility of Astatotilapia burtoni, a riverine species from the Lake Tanganyika basin, with Astatotilapia nubilus, a riverine species from the Lake Victoria basin. The fertility of the hybrids between these species suggests that they are genetically almost as compatible as haplochromines from the Lake Victoria basin $^{2,3}$. Moreover, we also tried to breed hybrids between the monotypic genus $A$ statoreochromis alluaudi and several haplochromines of Lake Victoria. According to Meyer et al., this species should be more closely related to Lake Victoria haplochromines than to those of Lake Tanganyika. We succeeded, however, in breeding only one hybrid population between Astatoreochromis alluaudi and Astatotilapia 'black lividus' (a so far undescribed species of Lake Victoria). These hybrids reproduced, but the eggs deteriorated before hatching. This is the only evidence for sterile hybrids that we have obtained so far. Astatoreochromis alluaudi is also the only species in Lake Victoria that stands out in its protein characteristics ${ }^{5}$.

We believe that Meyer et al. should include molecular data from the riverine Lake Tanganyika haplochromine $A$. burtoni in their analysis. Their second conclusion will then be that some haplochromines of different lakes arose from more closely related riverine species; in other words, that segregation had started before the lakes formed. The major problem of the species flocks of the great African lakes is clearly how they have evolved and are maintained in sympatry, while obviously relying exclusively on premating barriers - a significant ethological problem in its own right ${ }^{4}$.

M.-Domminique Crapon de Caprona

Berno FritzSch

Creighton University,

Department of Biomedical Sciences,

Omaha, Nebraska 68178, USA

MEYer ET Al. REPLY - Inferring relationships from patterns of hybridization is risky. The hybridization studies involving Astatotilapia burtoni males and Astatotilapia nubilis females produced only lethal hybrids ${ }^{2}$. Females of $A$. burtoni mated rarely with males of $A$. nubilis, but produced viable hybrids ${ }^{2}$. Numerous studies have shown that hybrid incompatibility, although usually correlated

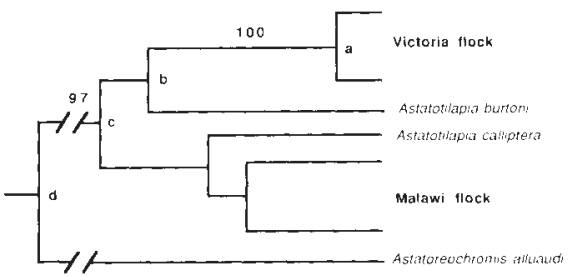

Evolutionary tree based on comparisons of part of the control region of mtDNA. The region sequenced and the methods used are described by Meyer et al. ${ }^{1}$. Bootstrap values appear above the two internal branches that are statistically significant $(a-b$ and $c-d)$. The distance from any node to the tips of the descendant branches are roughly proportional to the average number of base substitutional differences between species united by that node. The root of the tree (node d) was determined by using Julidochromis as an outgroup (see ref. 1). The two sequences of Astatotilapia have been deposited in Gen Bank with accession numbers X58151 (A.c.) and X58152 (A.b.).

with phylogenetic distance, is not an accurate metric for building trees and time scales.

In anticipation of questions about Astatotilapia's phylogenetic status, one of us (A. M.) sequenced the most variable part of the control region of mitochondrial DNA from two key species, $A$. burtoni and $A$. calliptera (a non-endemic cichlid from Lake Malawi). Three Astatotilapia species from the Lake Victoria basin (A. nubilis, A. elegans and $A$. piceatus) had already been tested ${ }^{1}$. The figure shows a tree relating the two new sequences to those of the species flocks in 
Lakes Victoria and Malawi as well as to Astatoreochromis alluaudi. The results confirm (with 97 per cent confidence) the hypothesis, suggested by interspecific hybridization studies, that $A$. burtoni is a closer relative of these flocks than is Astatoreochromis. This analysis also suggests (although not significant statistically) that $A$. burton $i$ is closer phylogenetically to the Lake Victoria flock than is the Lake Malawi flock.

Furthermore, and contrary to the hypothesis of Crapon de Caprona and Fritzsch above, the members of the Lake Victoria flock (including $A$. nubilis, $A$. elegans and $A$. piceatus are more closely related to one another mitochondrially than to $A$. burtoni. The bootstrap value that shows the reality of the $a-b$ lineage in the figure is 100 per cent. We infer that the Lake Victoria flock arose long after the splitting of the $A$. burtoni lineage from the $a-b$ lineage. Our molecular estimate of the time of most recent common ancestry for the Lake Victoria flock is 200,000 years ${ }^{1}$, which implies that the flock is younger than the lake $(250,000$ to 750,000 years old ${ }^{6}$ ). Thus, our results cast doubt on Crapon de Caprona and Fritzsch's model, above, according to which segregation occurred within the flock before the lake formed.

Another notable feature of the figure is that Astatotilapia species are candidates for being the sister groups of both the Lake Victoria and Lake Malawi flocks. Although Astatotilapia is not a monophyletic group (mitochondrially), these particular species may be close in body plan and lifestyle to the ancestral state for these two flocks, and this may help to explain their ability to hybridize with distant relatives.

Axel Meyer

Department of Ecology and Evolution,

State University of New York,

Stony Brook, NY 11794, USA

Department of Zoology,

THOMAS D. KOCHER

University of New Hampshire,

Durham, NH 03824, USA

Division of Biochemistry and

AlLan C. WILSON

Molecular Biology

University of California,

Berkeley, CA 94720, USA

1. Meyer, A., Kocher, T. D., Basasibwaki, P. \& Wilson, A. C. Nature 347, 550-553 (1990).

Crapon de Caprona, M.D. \& Fritzsch, B. Neth J Zool 34, $503-538(1984)$

3. Crapon de Caprona, M.-D. Ann. Sc. Zool. 251, 117-120 (1986a).

4. Crapon de Caprona, M.-D. J. Fish. Biol. 29, 151-158 (1986b).

5. Verheyen, E., Van der Linden, A. \& Decleir, W. Ann. Sc. Zool. 257, 93 (1989)

6. Temple, P. H. Biol. J. Linn. Soc. 1, 363-371 (1969). 